\title{
REVIEW
}

\section{Immune cell-mediated liver injury}

\author{
Nadia Corazza • Anastasia Badmann • Christoph Lauer
}

Received: 14 April 2009/Accepted: 27 May 2009/Published online: 17 June 2009

(C) Springer-Verlag 2009

\begin{abstract}
Liver diseases represent an important cause of morbidity and mortality in the world. Death of hepatocytes and other hepatic cell types is a characteristic feature of several forms of liver injury such as cholestasis, viral hepatitis, drug- or toxin-induced injury, and alcoholinduced liver damage. Moreover, irrespectively of the reason, liver injury seems to be facilitated by similar immune effector mechanisms common to these various liver diseases. Indeed, common immune effector mechanisms may explain the high prevalence of cirrhosis and cancer development in most forms of liver disease. Improved understanding of the immune cell-mediated mechanisms involved in hepatocyte cell death could be beneficial for the development of common therapeutic strategies against different forms of liver diseases. In this review, we will discuss novel findings on the role of different immune cells in liver disease and immune cellinduced death executioner mechanisms involved in hepatocyte cell death.
\end{abstract}

Keywords Liver disease · Tumor necrosis factor-related apoptosis-inducing ligand (TRAIL) - TNF family members . Tumor necrosis factor- $\alpha(\mathrm{TNF} \alpha) \cdot$ Fas ligand

\section{Introduction}

The liver represents a vital organ of the gastrointestinal system. Its main function is to take up nutrients, to store them, and to release them on demand to other organs. It

N. Corazza $(\bowtie) \cdot A$. Badmann $\cdot$ C. Lauer

Division of Immunopathology, Institute of Pathology,

University of Bern,

Murtenstrasse 31, P.O. Box 62, Bern 3010, Switzerland

e-mail: nadia.corazza@pathology.unibe.ch occupies a key position between the gastrointestinal and systemic venous circulations. One of its several functions is to filter the blood coming from the gut through the portal vein and from the systemic venous circulation through the liver artery. Because of its anatomical link to the intestinal tract and to the venous circulation, this organ is constantly exposed to several external compounds, potential pathogens, and food-derived antigens entering the body via the gut and drug metabolites, toxins, and tumor cells from the blood system. Thus, similar to the gut, the liver has developed, on one hand, a complex and particular immune system able to mount a rapid immune response against potentially dangerous pathogens without leading to damage of the organ and, on the other hand, complex apoptosis mechanisms of tissue cells, i.e., hepatocytes, to render them less susceptible to immune cell-mediated damage. Despite the constant exposure to low levels of gut-derived bacteria and bacterial products, no signs of ongoing inflammation are usually present in the liver under physiological condition. This lack of response is, to some extent, explained by very specific immunological properties of the liver, which contribute to a certain degree to systemic tolerance, as seen by graft survival, induction of systemic tolerance to gut-derived antigen, and persistence of some viral infections in the liver [1,2]. As in the gastrointestinal tract, the normal liver contains a large population of resident immune cells that differ from those in the peripheral blood. These cells include a large number of natural killer (NK) cells, NK T cells, Kupffer cells, and specific antigen-presenting cells. These specialized immune cells play an essential role in both liver immune homeostasis as well as in liver immunopathologies. For instance, NK $\mathrm{T}$ cells and NK cells participate in the suppression of liver metastases [3, 4]. Kupffer cells produce tumor necrosis factor- $\alpha(\mathrm{TNF} \alpha)$ and interleukin (IL)-10 in response to 
physiological concentration of lipopolysaccharide (LPS) [5-7].

The consequences of uncontrolled inflammatory immune responses have been illustrated during the pathogenesis of different liver diseases, such as viral hepatitis, alcoholic liver disease, and autoimmune hepatitis. For instance, upon viral infection of the liver virus-specific and bystander-activated $\mathrm{T}$ cells not only kill virus-infected cells but also cause severe damage to uninfected cells, leading to the development of liver injury. The specific role of immune cells and their effector mechanisms involved in different forms of liver injury has been extensively studied but is still a matter of discussion. NK cells, NK T cells, activated T cells, and Kupffer cells all play an essential role in different forms of liver pathology. Once activated, these immune cells express different tumor necrosis factor (TNF) family members, i.e., Fas ligand (FasL; CD95), TNF $\alpha$, and tumor necrosis factor-related apoptosisinducing ligand (TRAIL), potentially able to participate to hepatocyte cell death. Whether and how these cytotoxic mediators collaborate in hepatocyte apoptosis is still not sufficiently clear. One possibility could be that these mediators represent effector molecules of separate signaling pathways inducing hepatocyte cell death at different and independent checkpoints. The other possibility could be that these mediators represent effector molecules of the same apoptosis signaling cascade. Crosstalk between these apoptosis pathways will be discussed later.

Besides a complex immune system, the liver parenchymal cells, i.e., hepatocytes, have developed a peculiar and complex cell death machinery to render them less susceptible to potential dangerous signals. For instance, Fas receptor-induced apoptosis has been extensively studied in hepatocytes due to its critical dependence on the mitochondrial apoptosis pathway. In contrast to normal cells in which Fas triggering directly induces the activation of caspases and subsequently apoptosis (type I cells), hepatocytes require the amplification of the signal via the mitochondria (type II cells). While many tumor cells are type II cells, hepatocytes are probably the only known representatives of primary cells of this amplification loop of the Fas signaling pathways.

In this review, we will present an overview of the new findings on the role of death executioner mechanisms utilized by immune cells in liver disease. We will emphasize mainly the role of FasL, TNF $\alpha$, and TRAIL, respectively, and how these cytotoxic mediators collaborate in hepatocyte apoptosis in several forms of liver injury.

\section{Role of immune cell in liver pathologies}

Liver diseases are one of the most important causes of morbidity and mortality worldwide. Several different triggers have been implicated in the development of liver disease. These include hepatitis $\mathrm{B}$ and $\mathrm{C}$ virus, toxic compounds, alcohol abuse, and increase levels of free fatty acid. One of the major and common clinical problems of all of these forms of liver disease is the frequent development of liver cirrhosis or liver cancer related to chronic inflammation. Due to the limited availability of human liver samples and the difficulties to experimentally manipulate them, several animal models mimicking human liver diseases have emerged. The predominantly used animal models of liver disease are concanavalin A (ConA)-, LPS-, alcohol-, and polyinosinic/polycytidylic acid (Poly I:C)induced liver injury, and transgenic mouse models for hepatitis $\mathrm{B}$ and $\mathrm{C}$ virus. All of these models provide evidences that activation of inflammatory responses accounts for liver damage, as illustrated in Table 1. ConA, a plant-derived lectin that activates $\mathrm{T}$ lymphocytes and NK cells, has been widely exploited to induce acute liver injury in mice in a dose of $10-20 \mu \mathrm{g} / \mathrm{g}$ body weight [8]. In this model of liver disease, injury can be efficiently prevented by treating the mice with dexamethasone, an immunosuppressive steroid, indicating that liver disease is associated with the activation of immune cells [8]. Searching for these immune cell subsets responsible for ConA-induced liver damage, it was demonstrated that NK T cell-deficient mice $\left(\mathrm{CD} 1 \mathrm{~d}^{-/-}\right.$and $\mathrm{V} \alpha 14^{-/-}$mice) were resistant to this form of liver disease, suggesting that uncontrolled activation of these unconventional $\mathrm{T}$ cells, present in large numbers in the liver, plays a central role in the development of liver damage. However, not only NK T cells are essential in the development of liver disease but also several other cell subsets of the innate immune system, i.e., Kupffer cells, neutrophils, and eosinophils, have been shown to participate in the development of ConA-induced liver damage [912]. The exact mechanism how these different cell subsets interplay with each other in the development of liver injury is still not exactly known. Most probably, these immune cells either directly participate in liver cell death via the expression of apoptosis-related effector molecules, e.g., TNF $\alpha$, FasL, and TRAIL, or are involved in the amplification of the immune response via the release of proinflammatory mediators (e.g., interferon gamma (INF $\gamma$ ), IL-12, IL-1, IL-18, and chemokines) [13-17]. As summarized in Table 1, most of these cell populations not only represent relevant players in ConA-induced liver damage but also participate in the development of other forms of liver injury. For instance, Kupffer cells have been reported to be an essential initiator of pathologies in several hepatitis models. In the liver, activated Kupffer cells represent one of the major sources of proinflammatory cytokines, such as TNF $\alpha$ and IL-1 $\beta$. They constitutively express different tolllike receptor (TLRs), such as TLR3 and TLR4, on the cell surface and can be activated via the interaction of these 
Table 1 Most important immune effector cells and effector molecules involved in different forms of liver injury

\begin{tabular}{lll}
\hline Liver injury models & Effector cells & Effector molecules \\
\hline ConA & T cells (NK T cells) & INF $\gamma$, TNF $\alpha$, FasL, IL-4, TRAIL \\
& Kupffer cells & Superoxide anions, TNF $\alpha$ \\
& Neutrophils & \\
Kupffer cells & TNF $\alpha$, chemokines \\
Poly I:C & NK cells & FasL, TRAIL \\
Alcohol & NK T cells & FasL, TNF $\alpha$ \\
& Neutrophils & Chemokines \\
Free fatty acid & Kupffer cells & TNF $\alpha$, FasL \\
Paracetamol & NK cells & FasL, TNF $\alpha$, TRAIL \\
& Neutrophils & TNF $\alpha$ \\
\hline
\end{tabular}

receptors with their specific ligands Poly I:C and LPS, respectively [18]. Endogenous LPS is normally present at high concentrations in gram-negative bacteria colonizing the colon. Since nanograms of LPS injected into the blood stream can result in a vigorous inflammatory responses and, as a consequence, in septic shock, the body has developed strong defense mechanisms that tightly regulate the entry and processing of LPS to the body. Strategically located between the gastrointestinal tract and the portal blood flow, the liver plays a crucial role in this process, not only by its ability to clear LPS but also to respond vigorously to increased amount of LPS via the activation of Kupffer cells and the release of proinflammatory mediators and reactive oxygen intermediates. Endogenous gut-derived bacterial LPS has been indeed implicated as important cofactors in the pathogenesis of several models of liver disease. In alcohol- or carbon tetrachloride-induced liver injury, the damage was reduced in germ-free mice or after oral administration of antibiotics [19-21]. In contrast, additional LPS aggravated liver damage induced by these different hepatotoxic compounds [22]. In these models, the presence of LPS was responsible for the activation of Kupffer cells and, as a consequence, the release of TNF $\alpha$. Accordingly, inactivation of Kupffer cells with gadolinium chloride decreases injury in alcohol-induced liver disease [23]. Thus, given their location and their characteristics, Kupffer cells represent sentinel cells between the gastrointestinal tract and the systemic circulation able to mount a rapid immune response once a potential danger is entering the blood stream.

NK cells are another important player in the pathology of different liver diseases. This cell subset represents $20 \%$ of the lymphocytes population in the liver and has been considered a first line of defense against virus infection and liver metastases. Many groups have yet described the critical roles of NK cells in the pathogenesis of liver injury models, among which, Poly I:C-induced liver injury was the most useful for the understanding of the role of $\mathrm{NK}$ cells. In this model, activation of TLR3 via Poly I:C significantly induced accumulation and activation of $\mathrm{NK}$ cell in the liver [24, 25]. This form of liver injury was characterized by local spotty necrosis in the liver and only slight elevation of transaminase activity in the serum, in contrast to the massive necrotic lesions observed in ConAtreated animals. When analyzing how NK T cells induced liver damage, it was found that TRAIL, FasL, and perforin synergize in inducing hepatocyte cell death.

\section{Death receptors in liver pathology}

The continuously growing family of structurally related proteins of TNF family has received enormous scientific attention due to their important role in the regulation of cell activation, survival, death, and many other physiological and pathological events. All members of this family act as ligands and mediate their activity through cognate interaction with their respective transmembrane receptors. The ligands, including the prototypical members TNF $\alpha$, FasL, and TRAIL, have potent apoptosis-inducing activities in vivo and in vitro, indicating an important role for these molecules in various processes involving cellular suicide and cell-mediated cytotoxicity. The respective death receptors, interacting with these ligands, are transmembrane proteins with three major functional domains: an extracellular ligand-interacting domain, a transmembrane domain, and an intracellular death domain, which enable death receptors to initiate apoptosis signals. These death receptors include Fas, TNFR-1, and TRAIL receptors 1 and 2 . Concerning the liver, there is growing evidence demonstrating that apoptosis induced by the interaction of the ligands of TNF family member with the respective death receptors is a common feature in different forms of liver injury. Hepatocytes express all death receptors on their cell surface and can be activated by the respective ligands, which are present on several different activated immune cells [26]. 


\section{Fas receptor/ligand interaction in liver injury}

Among the death receptors, presumably the FasL/Fas system plays the most important pathogenic role in liver injury. Under physiological condition, hepatocytes as well as Kupffer cells, stellate cells, and endothelial cells express the Fas receptor on their cell surface, whereas the respective ligand, i.e., FasL, is normally expressed on NK cells and NK T cells. A physiological role of the Fas receptor in the liver was originally established in mice carrying natural mutation in the Fas receptor gene (lpr) [27, 28]. With increasing age, these mice develop liver hyperplasia demonstrating the need of the Fas receptor in maintaining tissue homeostasis [29]. Indeed, Fas-induced cell death represents an important mechanism for the removal of transformed hepatocytes, such as virus-infected cells or cancer cells, via activated NK $\mathrm{T}$ cells and NK cells. However, overactivation of the Fas receptor in the liver has been shown to lead to tissue destruction and organ failure. This has been well documented by massive hepatocyte apoptosis in mice after injection of activating anti-Fas antibodies or recombinant FasL [30, 31]. These mice died already 2-4 h after activation of the Fas receptor. Murine, as well as human hepatocytes, is, in fact, extremely sensitive to Fas-induced apoptosis, in vitro and in vivo. The intracellular mechanisms involved in this process will be discussed in details later. This Fas-induced pathway of hepatocyte death represents a common feature of several different forms of liver damage. Cell death induced by FasL/Fas interaction has been shown to play a central role not only in $\mathrm{T}$ cell-mediated liver pathologies induced by ConA and chronic viral hepatitis (e.g., hepatitis C) but also in liver injuries induced by toxic agents, such as acetaminophen overdose, alcohol, and cholestatic liver damage [32, 33]. Experiments using FasL-mutant mice ( $g l d$ ) or those using Fas knockdown by siRNA in mice provided clear evidence for a critical role of the Fas/FasL pathway in ConA-induced liver injury $[30,31]$. NK T cells expressing FasL were directly involved in the induction of hepatocyte apoptosis [34]. In humans, the role of FasL/Fas in the development of liver injury upon hepatitis $\mathrm{B}$ and $\mathrm{C}$ infection remains more enigmatic. In hepatitis $\mathrm{C}$ infection, upregulation of Fas on hepatocytes and FasL on activated $\mathrm{T}$ cells correlates with the severity of damage, and Fas expression was significantly higher on infected hepatocytes than on noninfected cells [35]. Fas expression could be induced in hepatocytes either by virus-specific proteins or by inflammatory cytokines such as IL-1 $\beta$ [36-39]. Activated cytotoxic $T$ cells expressing FasL were then able to induce apoptosis in virus-infected hepatocytes and also in neighboring uninfected hepatocytes, leading to tissue damage. On the other hand, it was demonstrated that the hepatitis $\mathrm{C}$ virus could protect hepatocytes from Fas- induced apoptosis by repressing the release of cytochrome c from the mitochondria [40]. Therefore, hepatitis virus proteins may both sensitize hepatocytes to Fas-induced apoptosis and inhibit apoptosis as a possible mechanism for viral immune escape and for maintaining persistent infection.

As mentioned earlier, FasL/Fas-mediated apoptosis plays an important role not only in direct $\mathrm{T}$ cell-mediated liver damage, such as during virus infections, but also in alcohol-, cholestasis-, as well as acetaminophen-induced liver damage [41]. Hepatocyte apoptosis has been reported to be an important feature of alcoholic hepatitis, and the number of apoptotic cells present in the affected liver correlates with the severity of disease, as hepatocyte apoptosis is enhanced in patients with high transaminase activity in the serum [38]. Similarly, enhanced expression of FasL and Fas has been observed in patients with alcoholic liver disease, compared to control individuals [42]. In cholestasis, elevated bile acid concentrations within the liver promote the development of liver cirrhosis and liver failure. Toxic bile acids induced hepatocytes death in vitro as well as in vivo by a Fas-dependent mechanism, and in agreement with these results, hepatocyte apoptosis was reduced in lpr and gld mice after bile duct ligation [43]. Thus, although the starting trigger of hepatocyte damage is completely different in these various liver diseases, the Fas-induced apoptosis pathway may represent a common apoptosis mechanism involved in several forms of liver injury.

\section{TNF receptor/ligand interactions in liver injury}

In addition to the FasL/Fas pathway, TNF $\alpha$ in its soluble as well as in its membrane-bound form has been demonstrated to participate in different forms of liver damage, including hepatitis, alcoholic liver injury, ischemia/reperfusion injury, and toxic liver damage [44]. TNF $\alpha$ is a pleiotropic proinflammatory cytokine produced in the liver mainly by activated Kupffer cells and in reduced amounts also by NK cells, endothelial cells, and stellate cells. TNF $\alpha$ can bind to two distinct receptors: TNFR-1 and TNFR-2. Both receptors are expressed on hepatocytes, although only TNFR-1 contains a so-called death domain and can directly execute apoptotic pathways. TNFR-2, on the other hand, does not contain a death domain, and its main function is to amplify the effect of TNFR-1 in promoting both cell death and inflammation [45]. In contrast to the Fas receptor, where the interaction with FasL has been shown to predominantly induce pro-apoptotic signals, activation of TNFR-1 induces both prosurvival signal as well as pro-apoptotic signals. Compared to Fas, TNFR signaling contains additional elements: Recruitment of the TNFR-associated protein (TRAF2) and receptor-interacting protein (RIP) leads to 
activation of $\mathrm{NF}-\mathrm{kB}$ and transcriptional activation of prosurvival genes (e.g., Bcl- $\mathrm{x}_{\mathrm{L}}, \mathrm{A} 1$, XIAP, and cellular FLICE-like inhibitory protein (cFLIP)). The fundamental role of TNFR-1 in the transcriptional activation of these prosurvival genes has been well documented in liver regeneration after partial hepatectomy [46]. TNFR-1, but not TNFR-2, plays an essential role in hepatocyte proliferation, via the activation of the NF- $\mathrm{KB}$ and STAT3 pathway [47]. Apoptotic signaling, on the other hand, occurs subsequently via the adaptor protein TNFR-associated death domain, which activates a Fas-associated death domain protein (FADD)/caspase 8-dependent pathway. Although TNFR-2 does not contain a death domain, both TNFR-1 and TNFR-2 were reported to collaborate in liver damage. The cooperative signaling of both receptors in liver disease has been attributed to the ability of the intracellular domain of TNFR-2 to attract adaptor molecules, such as TRAF2, thereby inhibiting TNFR-1-induced activation of the anti-apoptotic NF- $\mathrm{KB}$ pathway and in turn facilitating TNFR-1-induced activation of the pro-apoptotic machinery [48]. In this situation, the prosurvival signaling normally predominates. This is well demonstrated in TNF $\alpha$-mediated animal model of hepatitis, where inhibitors of protein synthesis need to be used in order to unmask the pro-apoptotic signals induced by $\mathrm{TNF} \alpha$ [49]. In addition, mice with a hepatocyte-specific deletion of either Ik kinase (IKK), IKK $\beta$, or IKK $\gamma$ are more sensitive to ConA- or LPS-induced liver disease [50].

It is currently clear that not only NF-KB plays a central role in the decision between hepatocyte survival and death but also the Jun kinase (JNK) pathway [51]. The role of JNK in liver disease has been investigated in several different models of injury. Whereas transient and modest activation of JNK by $\mathrm{TNF} \alpha$ is associated with cellular survival, prolonged and robust JNK activation contributes to hepatocyte cell death [52-54]. Moreover, the level of JNK activation seems to be controlled by NF- $\kappa \mathrm{B}$ activation. In the absence of NF- $\mathrm{BB}$ activation, TNF $\alpha$ constantly induces JNK activation. This extended activation of JNK contributes to cell death, and inhibition of JNK can rescue hepatocytes from $\mathrm{TNF} \alpha$ induced apoptosis in the presence of inhibitor of protein synthesis, such as actinomycin D [55, 56]. These results indicate that JNK exerts its pro-apoptotic activity independently of transcription. Similarly, pretreatment of mice with JNK inhibitors rescued the animals from the development of severe liver injury. Moreover, $\mathrm{TNF} \alpha$-induced activation of JNK correlated with liver damage after injection of ConA, and JNK inhibitors could block hepatocyte cell death induced by ConA $[57,58]$. In contrast to these observations, it was recently demonstrated that $\mathrm{JNK}$ is critically required for $\mathrm{TNF} \alpha$ expression in hematopoietic cells and it is not mandatory for TNF $\alpha$-triggered hepatocyte cell death during the development of liver damage [59].
The biological role of $\mathrm{TNF} \alpha$ in liver disease is even more complex because $\mathrm{TNF} \alpha$ plays an essential role as inflammatory cytokine in the activation and recruitment of different immune cells involved in liver disease, such as neutrophils, NK cells, and activated T cells, to the site of inflammation. Moreover, TNF $\alpha$ also mediates caspaseindependent death via the formation of reactive oxygen species. RIP-1 is recruited to TNFR-1 and activates Nox1 (reduced nicotinamide adenine dinucleotide phosphate oxidase), leading to reactive oxygen species formation, sustained activation of $\mathrm{JNK}$, and, as a consequence, hepatocyte cell death [60].

\section{TRAIL signaling pathways}

None of the apoptosis-inducing members of the TNF family has received as much attention in tumor biology as TRAIL. The reason for this strong interest is the fact that recombinant TRAIL specifically induces apoptosis in a broad range of tumor cells, while leaving normal nontransformed cells mostly unaffected. This is particularly important in the liver. Although under physiological conditions hepatocytes express TRAIL-Rs on their cell surface and the respective ligand, i.e., TRAIL, is normally expressed on hepatic NK cells and NK T cells, nontransformed hepatocytes seem not to be sensitive to TRAIL cytotoxicity [61, 62]. This finding has been of outmost interest since injection of recombinant TRAIL does not lead to liver damage, whereas recombinant FasL results in severe tissue destruction and even death [63, 64]. It has thus been suggested that nontransformed cells and, in particular, hepatocytes are not susceptible to TRAILinduced apoptosis, opening all possibilities for TRAILbased cancer therapy. In vitro as well as in vivo tumor models confirmed this idea. For instance, TRAIL treatment substantially inhibited growth of a variety of human tumors, including breast and colon carcinomas, gliomas, and multiple myelomas, in xenograft experiments in immunodeficient mice [65-68]. Critically, TRAIL treatment did not appear to have any side effects on normal tissue cells, in particular, in the liver. Moreover, Takeda et al. reported that TRAIL expressed on hepatic NK cells plays a substantial role in suppressing tumor metastasis without leading to hepatocyte damage [69]. They showed that freshly isolated NK cells from the liver expressed cell surface TRAIL, which was responsible for spontaneous cytotoxicity against TRAIL-sensitive tumor cells in vitro. Administration of neutralizing monoclonal antibodies against TRAIL significantly increased experimental liver metastases of several TRAIL-sensitive tumor cell lines [69]. These experiments indicate that TRAIL-mediated cytotoxicity of tumor cells by liver NK cells is an important 
antitumor mechanism, responsible for effective immune surveillance. Consequently, one would expect that lack of TRAIL leads to a dramatic increase in tumor incidence. Surprisingly, however, TRAIL-deficient mice develop normally and do not show spontaneous tumor development, as it has been observed in p53-deficient mice [70]. Nonetheless, endogenous TRAIL may still play an important role in the regulation of cancer development, since syngeneic renal cell carcinomas [71] and B cell lymphomas [70] grow faster and induce significantly more liver metastasis in TRAIL-deficient mice than in control animals.

Several groups have questioned the specificity of TRAIL-induced cell death for tumor cells, and accumulating evidence suggests now that TRAIL-induced apoptosis not only is limited to tumor cells but may also be induced in primary cells. It is likely that this observation has been missed previously since TRAIL sensitivity in primary cells may be under stringent control and only be activated under certain pathological conditions. For example, it was demonstrated that TRAIL might be involved in several forms of liver disease. Most notably, we and others demonstrated that TRAIL participates in ConA-induced liver disease ([72] and unpublished data). While these data convincingly show reduced liver damage in TRAILdeficient mice, the question remains why injection of recombinant TRAIL, in contrast to recombinant FasL, does not cause any signs of hepatitis. NK cells constitutively express TRAIL; however, cellular activation may result in increased cell surface expression of transmembrane TRAIL $[16,25]$, thus potentially promoting TRAIL-induced apoptosis in hepatocytes. Activation of liver-homing NK T cells and NK cells, however, also leads to a massive burst in INF $\gamma$ production. INF $\gamma$ is known to enhance the susceptibility of various cells to death receptor-induced apoptosis. In particular, Fas-induced apoptosis in tumor cells is amplified by pretreatment with INF $\gamma$. It is thus possible that high level of INF $\gamma$ may result in increased susceptibility of hepatocytes to TRAIL-induced apoptosis $[73,74]$. This suggests that although administration of recombinant TRAIL might be safe under physiological conditions, any kind of inflammatory context, e.g., bacterial or viral infections, with subsequent release of proinflammatory cytokines, may allow or enhance TRAIL-induced hepatotoxicity. The role of TRAIL in the development of liver injury has subsequently been confirmed in many different types of liver disease, i.e., hepatitis B and C infection, and cholestatic liver disease $[25,37,63,75,76]$. In accordance with these results, we recently demonstrated that TRAIL participates also in acetaminophen-induced liver disease (A. Badmann, N. Corazza, T. Brunner, unpublished data). Although a lot of effort has been done to understand how exactly TRAIL contributes to liver damage, the precise molecular mechanism remains a matter of debate. Sensitivity of hepatocytes to TRAIL-induced cell death has been associated primarily with increased surface expression of TRAIL-Rs on hepatocytes and TRAIL on hepatocytes and NK cells. Hepatitis B virus infection of human hepatocytes, as well as free fatty acid, has been reported to induce the expression of TRAIL-R1 in hepatocytes [77], targeting them to NK cell-mediated lysis. Accordingly, TRAIL was increased in the serum of patient with viral hepatitis, and viral infections in mice induced TRAIL expression in the liver [78]. Since functional surface expression of TRAIL has often been associated with stimulation by cytokines, such as INFs, the upregulation of TRAIL may be an important underlying reason for the successful use of INF $\alpha$ as an approved treatment option of patients chronically infected with hepatitis viruses. INF $\alpha$ treatment may, on one hand, sensitize hepatocytes to TRAIL-induced apoptosis [79] and, on the other hand, lead to increased TRAIL expression in liver-homing lymphocytes [80]. Thus, upregulation of TRAIL and TRAIL-Rs on hepatocytes and liver-homing lymphocytes could be an important mechanism to selectively eliminate virally infected hepatocytes. Recently, however, Malhi et al. demonstrated that TRAIL is also an important mediator of apoptosis in nonviral liver damage, such as free fatty acidinduced liver disease [81]. They showed that oleic acid induced upregulation of TRAIL-R2 on hepatocytes sensitizing them to TRAIL cytotoxicity. Upregulation of TRAIL and TRAIL-R, however, seems not to be the only mechanism involved in the sensitization of hepatocytes to TRAIL-induced cytotoxicity. It appears that sensitivity toward TRAIL-induced apoptosis does not always correlate with TRAIL receptor expression on target cells, but several other intracellular factors may play an essential role in rendering hepatocytes sensitive to TRAIL-induced cytotoxicity. For instance, it was demonstrated that hepatitis C virus infection sensitizes human hepatocytes to TRAILinduced apoptosis in a caspase 9-dependent manner [37, 82]. In line with these findings, other groups clearly showed that the hepatitis B virus sensitizes hepatocytes to TRAIL-induced apoptosis via the Bcl-2 protein Bax [83]. Taken together, all these results demonstrated that under particular inflammatory conditions, TRAIL directly induces hepatocyte apoptosis via interaction with its receptors. Interestingly, the very analogous trigger of liver disease has been demonstrated to sensitize hepatocytes not only to TRAIL but also to FasL- as well as TNF $\alpha$-induced cytotoxicity. For instance, we and others demonstrated that not only TRAIL but also other death ligands, such as TNF $\alpha$ and FasL, are crucial for lectin-induced liver damage and associated hepatitis. Indeed, it is well established that FasL is expressed on activated liver $\mathrm{T}$ and $\mathrm{NK} \mathrm{T}$ cells and contributes to liver damage in experimental hepatitis. These observations, together with the fact that injection of 
recombinant TRAIL does not cause any signs of hepatitis, suggested to us that TRAIL signaling, rather than directly inducing hepatocyte apoptosis, might cooperate with other death signals in promoting hepatocyte cell death in vivo. Thus, TRAIL may actually participate to hepatocyte apoptosis by modulating apoptosis induced by other triggers. In support of this notion, we found that recombinant TRAIL alone does not induce apoptosis in isolated murine hepatocytes; however, it strongly synergizes with FasL in promoting hepatocyte cell death [63]. More importantly, TRAIL-deficient mice were significantly more resistant to liver damage and associated death in response to injection of an agonistic anti-Fas antibody than control animals. Similarly, we observed that TRAIL participates to acetaminophen-induced liver damage (unpublished data). In the presence of TRAIL, human hepatocytes were more sensitive to acetaminophen-induced cell death, and TRAILdeficient mice were partially protected from the deleterious effect of acetaminophen overdoses in vivo. This observation confirms the results obtained by others, who demonstrated that deletion of NK cells, expressing TRAIL and FasL, has a beneficial effect on the pathology of acetaminophen-induced liver injury [84]. Taken together, these findings suggest that different death-inducing signals might collaborate in inducing hepatocyte apoptosis.

\section{Modulation of the hepatocyte apoptosis}

During the development of liver pathology, different apoptosis-inducing mediators contribute to the development of liver pathology and can influence the severity of the disease. However, it is still a matter of debate whether these apoptosis-inducing triggers, such as FasL, TRAIL, and $\mathrm{TNF} \alpha$, represent mediators of independent parallel pathways or whether these diverse death triggers interact with each other in order to amplify the apoptotic signal and to mount a stronger and more efficient cell death response. A complex control of hepatocyte apoptosis may be required to regulate cell death of this physiologically so important cell type. This would particularly make sense during the development of liver immunopathologies. For instance, during virus infection, activated immune cells, such as $\mathrm{T}$ cells or NK cells, simultaneously expresses FasL, TRAIL, and TNF $\alpha$ as well as perforin, which can cooperate in target cell killing. Our own data suggest that these different TNF family members do not initiate completely independent parallel pathways, but rather collaborate together in triggering hepatocyte apoptosis. In support to this notion, we found that recombinant TRAIL alone does not induce apoptosis in isolated murine hepatocytes; however, it strongly synergizes with FasL in promoting hepatocyte cell death [63].
Fas receptor-induced apoptosis has been extensively studied in hepatocytes due to its critical interaction with the mitochondrial apoptosis pathway. In contrast to normal cells in which Fas triggering directly induces the activation of caspases and subsequently apoptosis (type I cells), hepatocytes require the amplification of the signal via the mitochondria (type II cells). While many tumor cells are type II cells, hepatocytes are probably the only known representatives of this amplification loop of the Fas signaling pathway. Triggering of Fas results only in limited activation of caspase 8 in the death-inducing signaling complex, unable to further activate the caspase amplification cascade. Instead, caspase 8 must cleave the proapoptotic Bcl-2 homolog Bid. The carboxyl-terminal fragment of Bid (tBid) then translocates to the mitochondria, where it mediates the release of cytochrome $\mathrm{c}$ and other pro-apoptotic molecules. Consequently, Bid-deficient mice are largely resistant to anti-Fas- and FasL-induced liver damage and death $[85,86]$. This finding clearly demonstrates that in hepatocytes (and likely other type II cells), the Fas signaling pathway cannot be efficiently triggered in the absence of Bid, and thus, amplification of the death receptor apoptosis pathway via the intrinsic mitochondrial pathway is essential to induce hepatocyte cell death via the FasL/Fas pathway.

This amplification loop via the mitochondrial apoptosis pathway is not only restricted to FasL but represents also an essential mechanism in TNF $\alpha$-induced liver injury [49, 87]. Zhao et al. demonstrated that Bid-deficient mice are partially protected from LPS/D-galactosamine-induced liver injury proceeding via TNF/TNFR interactions [87]. Our own results, however, suggest that the apoptosis signaling cascade in this cell type may be even more complex and involves additional apoptosis effector molecules. We have observed that Fas-induced hepatocyte apoptosis and liver damage are critically controlled by TRAIL, JNK, and the pro-apoptotic Bcl-2 homolog Bim, as illustrated in Fig. 1 [63]. This additional control of apoptosis may add a further safe guard for the protection of these cells from accidental death induction and associated liver damage. TRAIL most likely participates in hepatocyte apoptosis by modulating the mitochondrial apoptosis pathway. While TRAIL alone fails to induce apoptosis, we clearly demonstrated that TRAIL modulated the FasL/Fas-induced apoptosis pathway via the activation of mitogen-activated protein kinases, in particular, activation of JNK resulting in the phosphorylation and activation of Bim. The precise mechanism how TRAIL activates JNK in hepatocytes is still only partially known. TRAIL-induced JNK activation occurs in a cell type-specific manner. As reported in other cell types, TRAIL can trigger JNK either through activation of FADD/caspase 8 or through the adaptor molecules, TRAF2 and/or RIP. 


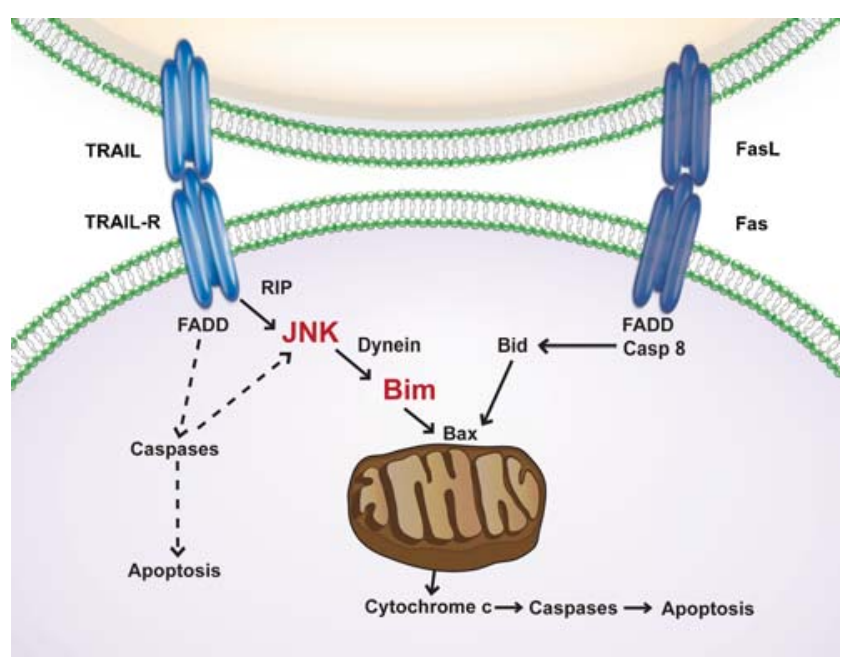

Fig. 1 Schematic model of the modulation of the Fas/FasL-induced apoptosis pathway by the TRAIL-JNK-Bim axis. While TRAIL alone fails to induce apoptosis, it triggers the activation of JNK. JNK leads to the phosphorylation of Bim and to its release from the cytoskeleton. Activation of Bim represents a second mitochondrial amplification loop, which further amplifies caspase-8- and Bid-initiated apoptosis signaling cascade in hepatocytes after Fas receptor ligation

As described above, JNK has been implicated in various forms of liver damage [53, 58, 59, 88]. We and others demonstrated a critical role of TRAIL in the activation of the JNK. For instance, we established that TRAIL activates JNK and thereby amplifies the Fas pathway. Similarly, Malhi et al. showed that free-fatty acids induce hepatocyte lipoapoptosis in a TRAIL- and JNK-dependent manner [81, 89]. Yet, it remains to be defined which JNK isoform is involved in TRAIL-induced modulation of hepatocyte apoptosis. Hepatocytes like most cell types express two JNK genes, $j n k 1$ and $j n k 2$. Their respective gene products, JNK1 and JNK2, both consist of two alternative splicing forms, p54 and p46 JNK. The p54 JNK isoform is predominantly expressed by $j n k 2$, and $\mathrm{p} 46 \mathrm{JNK}$ is primarily a jnkl product. Jnk1 and jnk2 null mice are both viable, whereas the double knockout mouse is embryonically lethal, suggesting that JNK1 and JNK2 have redundant activities. Recent studies, however, demonstrated that the products of $j n k 1$ and $j n k 2$ may have distinct functions. In cultured hepatocytes, bile acid toxicity is mediated by JNK2, whereas JNK1 is cytoprotective [89]. Similarly, it was demonstrated that $\mathrm{TNF} \alpha$-induced liver disease was JNK2-dependent [90]. Currently, it is unknown which JNK isoform is involved in TRAIL-induced modulation of hepatocyte cell death and how they regulate apoptosis. JNK1 and JNK2 could modulate apoptosis in several ways, including activation of pro-apoptotic Bcl-2 family proteins, inactivation of anti-apoptotic Bcl-2 family proteins, induction of BH-3-only proteins, degradation of the antiapoptotic molecule cFLIP, or modulation of death receptor expression. Searching for relevant JNK targets, we identi- fied Bim as a critical JNK-regulated response modifier in the TRAIL-initiated enhancement of hepatocyte apoptosis. JNK phosphorylates $\mathrm{Bim}_{\mathrm{EL}}$ and $\mathrm{Bim}_{\mathrm{L}}$ in vitro and in vivo, and in contrast to extracellular signal-related kinases 1 and 2-mediated phosphorylation and degradation by the proteosome, this leads to a dissociation of Bim from the cytoskeleton and its activation $[63,91]$. In agreement with this proposed pathway, we observed that inhibition of JNK and absence of TRAIL or Bim render mice largely resistant to anti-Fas-induced liver damage [63]. We thus propose that TRAIL-initiated activation of Bim represents a second mitochondrial amplification loop, which further amplifies Fas-induced caspase 8- and Bid-dependent apoptosis signaling in hepatocytes.

TRAIL-mediated enhancement of the mitochondrial pathway is also likely not restricted to Fas signaling in type II cells, such as hepatocytes, but may further extend to other triggers of the mitochondrial apoptosis pathway. In support of this idea, we found that TRAIL can modulate acetaminophen-induced liver damage in a JNK- and Bimdependent manner (unpublished data). Acetaminophen is a commonly used analgesic and antipyretic agent. Although considered safe at therapeutic doses, accidental or intentional overdose frequently causes acute liver failure characterized by centrilobular hepatic necrosis with high morbidity and mortality. Despite substantial efforts, the exact mechanisms of acetaminophen-induced liver cell injury remain incompletely understood. There is a general consensus that the P450 system-mediated formation of a toxic electrophilic metabolite, most probably $N$-acetyl $-p$ benzoquinone imine (NAPQI), is a prerequisite for acetaminophen-induced hepatocytes injury. NAPQI is usually detoxified by glutathione in the liver. However, acetaminophen overdose depletes hepatic glutathione inasmuch as NAPQI can covalently bind to cellular proteins leading to mitochondrial dysfunction. In experimental animal models, the progression and severity of acetaminophen-induced liver toxicity is not associated only with the induction of the membrane permeability transition and the collapse of mitochondrial membrane potential but depends also on the interplay of other death-inducing signals, both within and outside the hepatocytes. There is a significant overlap between events normally associated with apoptosis and acetaminophen-induced cell death. For instance, mitochondrial events generally linked to apoptosis have been observed in acetaminophen-induced hepatic cell death, including the truncation of Bid, Bax translocation to mitochondria, and release of cytochrome c [88, 92, 93]. In agreement with the role of pro-apoptotic molecules in this form of liver injury, we found that even in this type of liver damage, TRAIL, through the activation of the JNK and Bim axis, plays an essential role in acetaminophen-induced liver injury. Thus, we believed that TRAIL-mediated 
enhancement of the mitochondrial apoptosis pathway represents a general amplification mechanism involved in several models of hepatocyte cell death.

\section{Conclusion}

The question arises why TRAIL-induced signaling pathways in the liver are regulated in such a complex manner. As mentioned above, liver-homing NK and NK T cells are a rich source of TRAIL, and TRAIL-mediated cytotoxicity also seems to play an important role in the immune cellmediated control of liver metastases. Clearly, if hepatocytes would be intrinsically sensitive to TRAIL-induced apoptosis, the constitutive expression of TRAIL in liver NK and NK T cells would be a constant threat and likely lead to chronic liver destruction. Thus, it appears important that under normal conditions, hepatocytes are insensitive to TRAIL-induced apoptosis. The proposed amplification of the mitochondrial apoptosis pathway by the TRAIL-JNKBim axis may on the other hand ensure appropriate hepatocyte apoptosis in response to other triggers and under certain circumstances, e.g., when hepatocyte apoptosis is needed for the protection of the host. Of particular interest in this regard is the fact that viral infections render hepatocytes sensitive to TRAIL-induced apoptosis. Thus, TRAIL may represent a safeguard for the liver allowing the elimination of infected or transformed cells while sparing normal liver cells required for the vital functions of this important organ.

Acknowledgments Supported by grant from the Swiss National Science Foundation and Fondazione Ettore e Valeria Rossi (to N. Corazza).

\section{References}

1. Benseler V, McCaughan GW, Schlitt HJ, Bishop GA, Bowen DG, Bertolino P (2007) The liver: a special case in transplantation tolerance. Semin Liver Dis 27:194-213

2. Knolle PA (2006) Involvement of the liver in the induction of CD8 T cell tolerance towards oral antigen. Z Gastroenterol 44:5156

3. Bahjat KS, Prell RA, Allen HE, Liu W, Lemmens EE, Leong ML, Portnoy DA, Dubensky TW Jr, Brockstedt DG, Giedlin MA (2007) Activation of immature hepatic NK cells as immunotherapy for liver metastatic disease. J Immunol 179:7376-7384

4. Takeda K, Seki S, Ogasawara K, Anzai R, Hashimoto W, Sugiura K, Takahashi M, Satoh M, Kumagai K (1996) Liver NK1.1+ CD4+ alpha beta $\mathrm{T}$ cells activated by IL-12 as a major effector in inhibition of experimental tumor metastasis. J Immunol 156:33663373

5. Emmanuilidis K, Weighardt H, Maier S, Gerauer K, Fleischmann T, Zheng XX, Hancock WW, Holzmann B, Heidecke CD (2001) Critical role of Kupffer cell-derived IL-10 for host defense in septic peritonitis. J Immunol 167:3919-3927
6. Kurachi K, Suzuki S, Sakaguchi T, Yokoi Y, Konno H, Baba S, Nakamura S (2003) Kupffer cells modulate splenic interleukin-10 production in endotoxin-induced liver injury after partial hepatectomy. J Hepatol 38:193-199

7. Rai RM, Loffreda S, Karp CL, Yang SQ, Lin HZ, Diehl AM (1997) Kupffer cell depletion abolishes induction of interleukin10 and permits sustained overexpression of tumor necrosis factor alpha messenger RNA in the regenerating rat liver. Hepatology 25:889-895

8. Tiegs G, Hentschel J, Wendel A (1992) A T cell-dependent experimental liver injury in mice inducible by concanavalin A. J Clin Invest 90:196-203

9. Bonder CS, Ajuebor MN, Zbytnuik LD, Kubes P, Swain MG (2004) Essential role for neutrophil recruitment to the liver in concanavalin A-induced hepatitis. J Immunol 172:45-53

10. Hatada S, Ohta T, Shiratsuchi Y, Hatano M, Kobayashi Y (2005) A novel accessory role of neutrophils in concanavalin A-induced hepatitis. Cell Immunol 233:23-29

11. Hatano M, Sasaki S, Ohata S, Shiratsuchi Y, Yamazaki T, Nagata K, Kobayashi Y (2008) Effects of Kupffer cell-depletion on concanavalin A-induced hepatitis. Cell Immunol 251:25-30

12. Schumann J, Wolf D, Pahl A, Brune K, Papadopoulos T, van Rooijen N, Tiegs G (2000) Importance of Kupffer cells for T-celldependent liver injury in mice. Am J Pathol 157:1671-1683

13. Gantner F, Leist M, Lohse AW, Germann PG, Tiegs G (1995) Concanavalin A-induced T-cell-mediated hepatic injury in mice: the role of tumor necrosis factor. Hepatology 21:190-198

14. Kusters S, Gantner F, Kunstle G, Tiegs G (1996) Interferon gamma plays a critical role in T cell-dependent liver injury in mice initiated by concanavalin A. Gastroenterology 111:462-471

15. Kusters S, Tiegs G, Alexopoulou L, Pasparakis M, Douni E, Kunstle G, Bluethmann H, Wendel A, Pfizenmaier K, Kollias G, Grell M (1997) In vivo evidence for a functional role of both tumor necrosis factor (TNF) receptors and transmembrane TNF in experimental hepatitis. Eur J Immunol 27:2870-2875

16. Smyth MJ, Cretney E, Takeda K, Wiltrout RH, Sedger LM, Kayagaki N, Yagita H, Okumura K (2001) Tumor necrosis factorrelated apoptosis-inducing ligand (TRAIL) contributes to interferon gamma-dependent natural killer cell protection from tumor metastasis. J Exp Med 193:661-670

17. Tagawa Y, Kakuta S, Iwakura Y (1998) Involvement of Fas/Fas ligand system-mediated apoptosis in the development of concanavalin A-induced hepatitis. Eur J Immunol 28:4105-4113

18. Jiang W, Sun R, Wei H, Tian Z (2005) Toll-like receptor 3 ligand attenuates LPS-induced liver injury by down-regulation of tolllike receptor 4 expression on macrophages. Proc Natl Acad Sci U S A 102:17077-17082

19. Nolan JP (1975) The role of endotoxin in liver injury. Gastroenterology 69:1346-1356

20. Nolan JP (1989) Intestinal endotoxins as mediators of hepatic injuryan idea whose time has come again. Hepatology 10:887-891

21. Nolan JP, Camara DS (1989) Intestinal endotoxins as co-factors in liver injury. Immunol Invest 18:325-337

22. Luster MI, Germolec DR, Yoshida T, Kayama F, Thompson M (1994) Endotoxin-induced cytokine gene expression and excretion in the liver. Hepatology 19:480-488

23. Adachi Y, Bradford BU, Gao W, Bojes HK, Thurman RG (1994) Inactivation of Kupffer cells prevents early alcohol-induced liver injury. Hepatology 20:453-460

24. Dong Z, Wei H, Sun R, Hu Z, Gao B, Tian Z (2004) Involvement of natural killer cells in PolyI:C-induced liver injury. J Hepatol 41:966-973

25. Ochi M, Ohdan H, Mitsuta H, Onoe T, Tokita D, Hara H, Ishiyama K, Zhou W, Tanaka Y, Asahara T (2004) Liver NK cells expressing TRAIL are toxic against self hepatocytes in mice. Hepatology 39:1321-1331 
26. Malhi H, Gores GJ (2008) Cellular and molecular mechanisms of liver injury. Gastroenterology 134:1641-1654

27. Takahashi T, Tanaka M, Brannan CI, Jenkins NA, Copeland NG, Suda T, Nagata S (1994) Generalized lymphoproliferative disease in mice, caused by a point mutation in the Fas ligand. Cell 76:969-976

28. Watanabe-Fukunaga R, Brannan CI, Copeland NG, Jenkins NA, Nagata S (1992) Lymphoproliferation disorder in mice explained by defects in Fas antigen that mediates apoptosis. Nature 356:314-317

29. Adachi M, Suematsu S, Kondo T, Ogasawara J, Tanaka T, Yoshida N, Nagata S (1995) Targeted mutation in the Fas gene causes hyperplasia in peripheral lymphoid organs and liver. Nat Genet 11:294-300

30. Zender L, Hutker S, Liedtke C, Tillmann HL, Zender S, Mundt B, Waltemathe M, Gosling T, Flemming P, Malek NP, Trautwein C, Manns MP, Kuhnel F, Kubicka S (2003) Caspase 8 small interfering RNA prevents acute liver failure in mice. Proc Natl Acad Sci U S A 100:7797-7802

31. Ogasawara J, Watanabe-Fukunaga R, Adachi M, Matsuzawa A, Kasugai T, Kitamura Y, Itoh N, Suda T, Nagata S (1993) Lethal effect of the anti-Fas antibody in mice. Nature 364: 806-809

32. Batey RG, Wang J (2002) Molecular pathogenesis of $T$ lymphocyte-induced liver injury in alcoholic hepatitis. Front Biosci 7:d1662-d1675

33. Liu ZX, Han D, Gunawan B, Kaplowitz N (2006) Neutrophil depletion protects against murine acetaminophen hepatotoxicity. Hepatology 43:1220-1230

34. Kaneko Y, Harada M, Kawano T, Yamashita M, Shibata Y, Gejyo F, Nakayama T, Taniguchi M (2000) Augmentation of Valpha14 NKT cell-mediated cytotoxicity by interleukin 4 in an autocrine mechanism resulting in the development of concanavalin Ainduced hepatitis. J Exp Med 191:105-114

35. Hiramatsu N, Hayashi N, Katayama K, Mochizuki K, Kawanishi Y, Kasahara A, Fusamoto H, Kamada T (1994) Immunohistochemical detection of Fas antigen in liver tissue of patients with chronic hepatitis C. Hepatology 19:1354-1359

36. Bode JG, Brenndorfer ED, Haussinger D (2008) Hepatitis C virus (HCV) employs multiple strategies to subvert the host innate antiviral response. Biol Chem 389:1283-1298

37. Chou AH, Tsai HF, Wu YY, Hu CY, Hwang LH, Hsu PI, Hsu PN (2005) Hepatitis C virus core protein modulates TRAIL-mediated apoptosis by enhancing Bid cleavage and activation of mitochondria apoptosis signaling pathway. J Immunol 174:2160-2166

38. Iio S, Hayashi N, Mita E, Ueda K, Mochizuki K, Hiramatsu N, Kanto T, Sasaki Y, Kasahara A, Hori M (1998) Serum levels of soluble Fas antigen in chronic hepatitis $\mathrm{C}$ patients. J Hepatol 29:517-523

39. Pianko S, Patella S, Ostapowicz G, Desmond P, Sievert W (2001) Fas-mediated hepatocyte apoptosis is increased by hepatitis $\mathrm{C}$ virus infection and alcohol consumption, and may be associated with hepatic fibrosis: mechanisms of liver cell injury in chronic hepatitis $\mathrm{C}$ virus infection. J Viral Hepat 8:406-413

40. Sacco R, Tsutsumi T, Suzuki R, Otsuka M, Aizaki H, Sakamoto S, Matsuda M, Seki N, Matsuura Y, Miyamura T, Suzuki T (2003) Antiapoptotic regulation by hepatitis $\mathrm{C}$ virus core protein through up-regulation of inhibitor of caspase-activated DNase. Virology 317:24-35

41. Minagawa M, Deng Q, Liu ZX, Tsukamoto H, Dennert G (2004) Activated natural killer $\mathrm{T}$ cells induce liver injury by Fas and tumor necrosis factor-alpha during alcohol consumption. Gastroenterology 126:1387-1399

42. Tagami A, Ohnishi H, Moriwaki H, Phillips M, Hughes RD (2003) Fas-mediated apoptosis in acute alcoholic hepatitis. Hepatogastroenterology 50:443-448
43. Miyoshi H, Rust C, Roberts PJ, Burgart LJ, Gores GJ (1999) Hepatocyte apoptosis after bile duct ligation in the mouse involves Fas. Gastroenterology 117:669-677

44. Hatano E (2007) Tumor necrosis factor signaling in hepatocyte apoptosis. J Gastroenterol Hepatol 22(Suppl 1):S43-S44

45. MacEwan DJ (2002) TNF receptor subtype signalling: differences and cellular consequences. Cell Signal 14:477-492

46. Yamada Y, Kirillova I, Peschon JJ, Fausto N (1997) Initiation of liver growth by tumor necrosis factor: deficient liver regeneration in mice lacking type I tumor necrosis factor receptor. Proc Natl Acad Sci U S A 94:1441-1446

47. Yamada Y, Webber EM, Kirillova I, Peschon JJ, Fausto N (1998) Analysis of liver regeneration in mice lacking type 1 or type 2 tumor necrosis factor receptor: requirement for type 1 but not type 2 receptor. Hepatology 28:959-970

48. Costelli P, Aoki P, Zingaro B, Carbo N, Reffo P, Lopez-Soriano FJ, Bonelli G, Argiles JM, Baccino FM (2003) Mice lacking TNFalpha receptors 1 and 2 are resistant to death and fulminant liver injury induced by agonistic anti-Fas antibody. Cell Death Differ 10:997-1004

49. Kaufmann T, Jost PJ, Pellegrini M, Puthalakath H, Gugasyan R, Gerondakis S, Cretney E, Smyth MJ, Silke J, Hakem R, Bouillet P, Mak TW, Dixit VM, Strasser A (2009) Fatal hepatitis mediated by tumor necrosis factor TNFalpha requires caspase- 8 and involves the BH3-only proteins Bid and Bim. Immunity 30:56-66

50. Luedde T, Heinrichsdorff J, de Lorenzi R, De Vos R, Roskams T, Pasparakis M (2008) IKK1 and IKK2 cooperate to maintain bile duct integrity in the liver. Proc Natl Acad Sci U S A 105:97339738

51. Wullaert A, Heyninck K, Beyaert R (2006) Mechanisms of crosstalk between TNF-induced NF-kappaB and JNK activation in hepatocytes. Biochem Pharmacol 72:1090-1101

52. Javelaud D, Besancon F (2001) NF-kappa B activation results in rapid inactivation of JNK in TNF alpha-treated Ewing sarcoma cells: a mechanism for the anti-apoptotic effect of NF-kappa B. Oncogene 20:4365-4372

53. Tang G, Minemoto Y, Dibling B, Purcell NH, Li Z, Karin M, Lin A (2001) Inhibition of JNK activation through NF-kappaB target genes. Nature 414:313-317

54. De Smaele E, Zazzeroni F, Papa S, Nguyen DU, Jin R, Jones J, Cong R, Franzoso G (2001) Induction of gadd45beta by NFkappaB downregulates pro-apoptotic JNK signalling. Nature 414:308-313

55. Schwabe RF, Uchinami H, Qian T, Bennett BL, Lemasters JJ, Brenner DA (2004) Differential requirement for c-Jun NH2terminal kinase in TNF-alpha- and Fas-mediated apoptosis in hepatocytes. FASEB J 18:720-722

56. Marderstein EL, Bucher B, Guo Z, Feng X, Reid K, Geller DA (2003) Protection of rat hepatocytes from apoptosis by inhibition of c-Jun N-terminal kinase. Surgery 134:280-284

57. Ni HM, Chen X, Shi YH, Liao Y, Beg AA, Fan J, Yin XM (2009) Genetic delineation of the pathways mediated by bid and JNK in tumor necrosis factor-alpha-induced liver injury in adult and embryonic mice. J Biol Chem 284:4373-4382

58. Chang L, Kamata H, Solinas G, Luo JL, Maeda S, Venuprasad K, Liu YC, Karin M (2006) The E3 ubiquitin ligase itch couples JNK activation to TNF-alpha-induced cell death by inducing c-FLIP(L) turnover. Cell 124:601-613

59. Das M, Sabio G, Jiang F, Rincon M, Flavell RA, Davis RJ (2009) Induction of hepatitis by JNK-mediated expression of TNF-alpha. Cell 136:249-260

60. Kim YS, Morgan MJ, Choksi S, Liu ZG (2007) TNF-induced activation of the Nox1 NADPH oxidase and its role in the induction of necrotic cell death. Mol Cell 26:675-687

61. Guo Y, Chen C, Zheng Y, Zhang J, Tao X, Liu S, Zheng D, Liu Y (2005) A novel anti-human DR5 monoclonal antibody with 
tumoricidal activity induces caspase-dependent and caspaseindependent cell death. J Biol Chem 280:41940-41952

62. Ichikawa K, Liu W, Zhao L, Wang Z, Liu D, Ohtsuka T, Zhang H, Mountz JD, Koopman WJ, Kimberly RP, Zhou T (2001) Tumoricidal activity of a novel anti-human DR5 monoclonal antibody without hepatocyte cytotoxicity. Nat Med 7:954-960

63. Corazza N, Jakob S, Schaer C, Frese S, Keogh A, Stroka D, Kassahn D, Torgler R, Mueller C, Schneider P, Brunner T (2006) TRAIL receptor-mediated JNK activation and Bim phosphorylation critically regulate Fas-mediated liver damage and lethality. J Clin Invest 116:2493-2499

64. Walczak H, Miller RE, Ariail K, Gliniak B, Griffith TS, Kubin M, Chin W, Jones J, Woodward A, Le T, Smith C, Smolak P, Goodwin RG, Rauch CT, Schuh JC, Lynch DH (1999) Tumoricidal activity of tumor necrosis factor-related apoptosis-inducing ligand in vivo. Nat Med 5:157-163

65. Fandy TE, Shankar S, Srivastava RK (2008) Smac/DIABLO enhances the therapeutic potential of chemotherapeutic drugs and irradiation, and sensitizes TRAIL-resistant breast cancer cells. Mol Cancer 7:60

66. Sugamura K, Gibbs JF, Belicha-Villanueva A, Andrews C, Repasky EA, Hylander BL (2008) Synergism of CPT-11 and Apo2L/TRAIL against two differentially sensitive human colon tumor xenografts. Oncology 74:188-197

67. Fulda S, Debatin KM (2006) 5-Aza-2'-deoxycytidine and IFNgamma cooperate to sensitize for TRAIL-induced apoptosis by upregulating caspase-8. Oncogene 25:5125-5133

68. Mitsiades CS, Treon SP, Mitsiades N, Shima Y, Richardson P, Schlossman R, Hideshima T, Anderson KC (2001) TRAIL/Apo2L ligand selectively induces apoptosis and overcomes drug resistance in multiple myeloma: therapeutic applications. Blood 98:795-804

69. Takeda K, Hayakawa Y, Smyth MJ, Kayagaki N, Yamaguchi N, Kakuta S, Iwakura Y, Yagita H, Okumura K (2001) Involvement of tumor necrosis factor-related apoptosis-inducing ligand in surveillance of tumor metastasis by liver natural killer cells. Nat Med 7:94-100

70. Sedger LM, Glaccum MB, Schuh JC, Kanaly ST, Williamson E, Kayagaki N, Yun T, Smolak P, Le T, Goodwin R, Gliniak B (2002) Characterization of the in vivo function of TNF-alpharelated apoptosis-inducing ligand, TRAIL/Apo2L, using TRAIL/ Apo2L gene-deficient mice. Eur J Immunol 32:2246-2254

71. Seki N, Hayakawa Y, Brooks AD, Wine J, Wiltrout RH, Yagita H, Tanner JE, Smyth MJ, Sayers TJ (2003) Tumor necrosis factorrelated apoptosis-inducing ligand-mediated apoptosis is an important endogenous mechanism for resistance to liver metastases in murine renal cancer. Cancer Res 63:207-213

72. Zheng SJ, Wang P, Tsabary G, Chen YH (2004) Critical roles of TRAIL in hepatic cell death and hepatic inflammation. J Clin Invest 113:58-64

73. Tagawa Y, Sekikawa K, Iwakura Y (1997) Suppression of concanavalin A-induced hepatitis in IFN-gamma(-l-) mice, but not in TNF-alpha $(-/-)$ mice: role for IFN-gamma in activating apoptosis of hepatocytes. J Immunol 159:1418-1428

74. Wang S, Boonman ZF, Li HC, He Y, Jager MJ, Toes RE, Niederkorn JY (2003) Role of TRAIL and IFN-gamma in CD4+ T cell-dependent tumor rejection in the anterior chamber of the eye. J Immunol 171:2789-2796

75. Kahraman A, Barreyro FJ, Bronk SF, Werneburg NW, Mott JL, Akazawa Y, Masuoka HC, Howe CL, Gores GJ (2008) TRAIL mediates liver injury by the innate immune system in the bile duct-ligated mouse. Hepatology 47:1317-1330

76. Mundt B, Kuhnel F, Zender L, Paul Y, Tillmann H, Trautwein C, Manns MP, Kubicka S (2003) Involvement of TRAIL and its receptors in viral hepatitis. FASEB J 17:94-96

77. Janssen HL, Higuchi H, Abdulkarim A, Gores GJ (2003) Hepatitis $\mathrm{B}$ virus enhances tumor necrosis factor-related apoptosis-inducing ligand (TRAIL) cytotoxicity by increasing TRAIL-R1/death receptor 4 expression. J Hepatol 39:414-420

78. Chen GY, He JQ, Lu GC, Li MW, Xu CH, Fan WW, Zhou C, Chen Z (2005) Association between TRAIL expression on peripheral blood lymphocytes and liver damage in chronic hepatitis B. World J Gastroenterol 11:4090-4093

79. Shigeno M, Nakao K, Ichikawa T, Suzuki K, Kawakami A, Abiru S, Miyazoe S, Nakagawa Y, Ishikawa H, Hamasaki K, Nakata K, Ishii N, Eguchi K (2003) Interferon-alpha sensitizes human hepatoma cells to TRAIL-induced apoptosis through DR5 upregulation and NF-kappa B inactivation. Oncogene 22:1653-1662

80. Sato K, Hida S, Takayanagi H, Yokochi T, Kayagaki N, Takeda K, Yagita H, Okumura K, Tanaka N, Taniguchi T, Ogasawara K (2001) Antiviral response by natural killer cells through TRAIL gene induction by IFN-alpha/beta. Eur J Immunol 31:3138-3146

81. Malhi H, Barreyro FJ, Isomoto H, Bronk SF, Gores GJ (2007) Free fatty acids sensitise hepatocytes to TRAIL mediated cytotoxicity. Gut 56:1124-1131

82. Lan L, Gorke S, Rau SJ, Zeisel MB, Hildt E, Himmelsbach K, Carvajal-Yepes M, Huber R, Wakita T, Schmitt-Graeff A, Royer C, Blum HE, Fischer R, Baumert TF (2008) Hepatitis C virus infection sensitizes human hepatocytes to TRAIL-induced apoptosis in a caspase 9-dependent manner. J Immunol 181:4926-4935

83. Liang X, Liu Y, Zhang Q, Gao L, Han L, Ma C, Zhang L, Chen YH, Sun W (2007) Hepatitis B virus sensitizes hepatocytes to TRAIL-induced apoptosis through Bax. J Immunol 178:503-510

84. Liu ZX, Govindarajan S, Kaplowitz N (2004) Innate immune system plays a critical role in determining the progression and severity of acetaminophen hepatotoxicity. Gastroenterology 127:1760-1774

85. Kaufmann T, Tai L, Ekert PG, Huang DC, Norris F, Lindemann RK, Johnstone RW, Dixit VM, Strasser A (2007) The BH3-only protein bid is dispensable for DNA damage- and replicative stressinduced apoptosis or cell-cycle arrest. Cell 129:423-433

86. Yin XM, Wang K, Gross A, Zhao Y, Zinkel S, Klocke B, Roth KA, Korsmeyer SJ (1999) Bid-deficient mice are resistant to Fasinduced hepatocellular apoptosis. Nature 400:886-891

87. Zhao Y, Li S, Childs EE, Kuharsky DK, Yin XM (2001) Activation of pro-death Bcl-2 family proteins and mitochondria apoptosis pathway in tumor necrosis factor-alpha-induced liver injury. J Biol Chem 276:27432-27440

88. Hanawa N, Shinohara M, Saberi B, Gaarde WA, Han D, Kaplowitz N (2008) Role of JNK translocation to mitochondria leading to inhibition of mitochondria bioenergetics in acetaminophen-induced liver injury. J Biol Chem 283:13565-13577

89. Malhi H, Bronk SF, Werneburg NW, Gores GJ (2006) Free fatty acids induce JNK-dependent hepatocyte lipoapoptosis. J Biol Chem 281:12093-12101

90. Wang Y, Singh R, Lefkowitch JH, Rigoli RM, Czaja MJ (2006) Tumor necrosis factor-induced toxic liver injury results from JNK2-dependent activation of caspase- 8 and the mitochondrial death pathway. J Biol Chem 281:15258-15267

91. Ley R, Ewings KE, Hadfield K, Cook SJ (2005) Regulatory phosphorylation of Bim: sorting out the ERK from the JNK. Cell Death Differ 12:1008-1014

92. El-Hassan H, Anwar K, Macanas-Pirard P, Crabtree M, Chow SC, Johnson VL, Lee PC, Hinton RH, Price SC, Kass GE (2003) Involvement of mitochondria in acetaminophen-induced apoptosis and hepatic injury: roles of cytochrome c, Bax, Bid, and caspases. Toxicol Appl Pharmacol 191:118-129

93. Nakagawa H, Maeda S, Hikiba Y, Ohmae T, Shibata W, Yanai A, Sakamoto K, Ogura K, Noguchi T, Karin M, Ichijo H, Omata M (2008) Deletion of apoptosis signal-regulating kinase 1 attenuates acetaminophen-induced liver injury by inhibiting c-Jun N-terminal kinase activation. Gastroenterology 135:1311-1321 